\title{
NUEVO HALLAZGO DE TARASA UMBELLATA KRAPOV. (MALVACEAE)
}

\section{NEW DISCOVERY OF TARASA UMBELLATA KRAPOV.(MALVACEAE)}

\author{
Alicia Marticorena ${ }^{1}$, José Antonio Valdivieso ${ }^{2} \&$ Carlos M. Baeza $^{1}$ \\ ${ }^{1}$ Departamento de Botánica, Universidad de Concepción, Casilla 160-C, Concepción, Chile; ${ }^{2}$ Carlos Antúnez 2185 , \\ Dpto. 22, Providencia, Santiago, Chile. \\ amartic@udec.cl
}

\section{RESUMEN}

Se presenta el nuevo hallazgo de Tarasa umbellata Krapov. (Malvaceae) en Talca, Chile. Los frutos eran desconocidos hasta ahora. Se entrega una descripción completa, comentarios de su hábitat, número cromosómico y la relación con Tarasa reichei (Phil.) Krapov.

\section{ABSTRACT}

A new discovery of Tarasa umbellata Krapov. (Malvaceae) in Talca, central Chile, is presented. The fruits were unknown until now. The description of the fruits, commentaries about the habitat, chromosome number and the relation with Tarasa reichei (Phil.) Krapov. is made.

\section{INTRODUCCION}

El género Tarasa Phil. posee 30 especies, que crecen desde el centro de Perú hasta la zona central de Chile y Argentina (Tate 2003a), se ha dividido en dos secciones, Tarasa y Umbellata (Krapovickas 1960). En Chile se encuentran 10 especies (Marticorena 2005), desde los $18^{\circ} 12^{\prime}$ a los $38^{\circ} 47^{\prime}$ de latitud sur, la mayoría entre los 1150 y 3800 m de altitud, excepto las especies de la sección Umbellata, T. umbellata Krapov. y T. reichei (Phil.) Krapov. (Krapovickas 1960), ambas muy escasas, y de altitudes más bajas.

Krapovickas (1960) describe T. umbellata de un material recolectado por Luis Sanfurgo, depositado en el Herbario del Museo Nacional de Historia Natural de Santiago de Chile (F. Philippi No 1960), espécimen sólo con flores, por lo que los frutos eran desconocidos.

Luego de más de 100 años, se vuelve encontrar $T$. umbellata, en áreas cercanas a su ubicación original, en el sector rural conocido como "Puertas Negras", distante unos $8 \mathrm{~km}$ al SE de la ciudad de Talca, dentro de la propiedad conocida como "Parcela La Zunilda" (35²8'48"S/71³6'05"W), a $130 \mathrm{~m}$ de altitud, y que corresponde a un remanente de bosque nativo higrofítico que flanquea a un estero conocido localmente como "Aguas Negras". Este bosque ha permanecido con poca alteración dado que el suelo es altamente húmedo para la actividad agrícola y por la dificultad de acceso, debido a la gran cantidad de Rubus ulmifolius Schott (zarzamora). El lugar es usado por los campesinos para la extracción ocasional de postes para cercos y como lugar de cacería de aves. En sus márgenes existe una zona de vega con vegetación característica de suelos inundados, donde se encuentran representantes de los géneros Scirpus, Juncus y Mimulus, entre otros. Hacia el interior se encuentran árboles de hasta unos $12 \mathrm{~m}$ de alto, siendo las especies predominantes Drimys winteri J.R.Forst. et G.Forst., Crinodendron patagua Molina, Maytenus boaria Molina y diversas 
mirtáceas, destacando por su abundancia Luma chequen (Molina) A.Gray, Aristotelia chilensis (Molina) Stuntz y Myrceugenia exsucca (DC.) O.Berg. Las trepadoras nativas están representadas por Lardizabala biternata Ruiz et Pav., Boquila trifoliolata (DC.) Decne., Cissus striata Ruiz et Pav., Bomarea salsilla (L.) Herb. Entre la vegetación introducida, además del ya mencionado Rubus ulmifolius, se encuentra la presencia de algunos individuos de Salix babilonica Willd., Populus nigra L. var. italica Münchh. y Acacia melanoxylon R.Br.

Los individuos de Tarasa umbellata se ubican en un pequeño claro del bosque formado por la caída de un árbol. La superficie estimada donde se presentan numerosos ejemplares es de unos $7 \times 7 \mathrm{~m}$. Estos nuevos ejemplares en la primera recolección se encontraban en flor, mientras, que en la segunda oportunidad los mismos ya presentaban frutos en un estado avanzado de maduración, lo que permite completar su descripción.

Debido a que se pudo obtener frutos con semillas viables, se realizó un estudio del número de cromosomas de la especie, el cual permite reafirmar la división que Krapovickas (1960) hace del género.

\section{MATERIALES Y METODOS}

Se estudiaron materiales de los herbarios CONC y SGO, y se compararon con las descripciones originales y los materiales tipos. Se tomaron fotos de los especímenes en terreno y detalles de los frutos en el laboratorio.

Para el estudio de los cromosomas se utilizó la metodología propuesta por Weiss-Schneeweiss et al. (2007). Los cromosomas (10 placas metafásicas) se clasificaron de acuerdo a Levan et al. (1964, modificado) y se midieron con el programa “MicroMeasure 3.3', (Reeves 2001). Para la confección del idiograma se utilizó el programa Corel Draw 8.0.

\section{MATERIALES ESTUDIADOS}

\section{Tarasa umbellata:}

Chile. VII Región, Prov. Curicó, L. Sanfurgo s/n, Herbario F.Philippi 1960 (Tipo: SGO 41062); Prov. Talca, Puertas Negras, ca. $8 \mathrm{~km}$ al SE de la ciudad de Talca, Parcela La Zunilda, (35²8'48', S/
71'36'05',W), a 130 m, 30-X-2006, J.A. Valdivieso (CONC 163805), 24-XII-2006, J.A. Valdivieso (CONC 163806).

\section{Tarasa reichei:}

Chile. VII Región. Prov. Cardenal Caro. San Pedro de Alcántara, 70 m, 29. X. 2005, N.García 1431 (CONC 163626). VII Región. Prov. Talca, Constitución. Reiche s/n (Tipo SGO 51946).

\section{RESULTADOS}

\section{DESCRIPCIÓN}

Arbustos pequeños formados por tallos delgados, de hasta $2 \mathrm{~cm}$ de diámetro, flexibles y arqueados que crecen formando una red intrincada entre la vegetación, apoyándose muchas veces directamente sobre el suelo, o bien se encuentran semienterrados. Ramas de hasta $2,5 \mathrm{~m}$ de alto en las zonas abiertas, alcanzando hasta $6 \mathrm{~m}$ aproximadamente cuando se apoyan en árboles grandes, totalmente cubiertas de pelos estrellados de ramas largas y numerosas. Pecíolo de 2-3,5 cm, cubierto de pelos estrellados de ramas largas y numerosas. Lámina de tamaño y forma variables, dependiendo de la ubicación en la planta, de forma triangular-cordada, algunas con tendencia a ser trilobadas, de 5,5-10,4 x 3,6-6,3 cm (Fig. 1A), margen irregularmente crenado, ambas caras con pelos estrellados, de 2 hasta cerca de 10 ramas, Inflorescencia axilar umbeliforme, con 7-9 flores de color blanco, generalmente más larga que la hoja que la subtiende. Pedúnculos florales de 4,5-7 cm de largo. Flores con 3 brácteas, linear-lanceoladas, de $4 \mathrm{~mm}$ (Fig. 1B). Cáliz de 4,5-5 mm de alto, formado por 5 sépalos unidos hasta cerca de la mitad del largo, lóbulos triangulares, agudos, cara externa con pelos estrellados, cara interna glabra. Nectarios en la base de los sépalos, formando un anillo, de 0,2-0,5 mm de alto. Pétalos 5, obovados, de $6 \mathrm{~mm}$ de largo. Estambres numerosos. Ovario cubierto de pelos estrellados, estilos 10, de casi el largo de los estambres, estigmas capitados (Fig. 1C). Frutos esquizocárpicos son abundantes en las ramas terminales más altas, formados por hasta 12 mericarpos (por subdivisión de algún lóculo (Stewart 2006)), de contorno redondeadoreniforme, con una breve escotadura en la cara abaxial, cubierta de pelos estrellados, con una 
arista de 2,5-4,5 mm de largo, curvada hacia atrás, con pelos estrellados de ramas largas y numerosas (Fig. 1 D, E). Semillas reniformes, de 1 x 1,5 mm, de color pardo, glabras, opacas.

ANÁLISIS CROMOSÓMICO

La muestra analizada presenta una dotación cromosómica $2 \mathrm{n}=2 \mathrm{x}=12$ y una fórmula cromosomal
$5 \mathrm{~m}+1 \mathrm{sm}$, esto es, cinco pares de cromosomas metacéntricos y un par submetacéntrico (Figs. 2 y 3 ).

\section{OBSERVACIONES}

Tarasa reiche, la otra especie de la sección Umbellata, es muy similar a T. umbellata, pero se puede diferenciar por el largo de la inflorescencia, y en el tamaño y forma del fruto (Fig. 1F).

\section{Clave Para diferenciar las especies de la SeCción UMbella Ta}

1. Inflorescencia en general más corta que la hoja que la subtiende; parte basal de los mericarpos de $1,5 \times 1,5$ $\mathrm{mm}$, con arista recta de 1,5-2 $\mathrm{mm}$ (Fig. $1 \mathrm{Fa}$ ).

1. Inflorescencia en general de igual largo o más larga que la hoja que la subtiende; parte basal de los mericarpos de 2 x 2-2,5 mm, con arista curva de 2,5-4,5 mm (Fig. $1 \mathrm{Fb}$ )

T. umbellata

\section{DISCUSION}

Respecto del número cromosómico, Krapovickas (1960) indica para 15 especies de Tarasa la presencia de 8 diploides $(2 n=10)$ y 7 tetraploides $(2 n=20)$, señalando que este género presenta un número básico $\mathrm{x}=5$, con la presencia casi constante de al menos un par de cromosomas con satélites. La población estudiada de Tarasa umbellata presenta una dotación cromosómica $2 \mathrm{n}=12$, lo que no se corresponde con lo señalado anteriormente, además de la ausencia de cromosomas con satélites. Este nuevo número cromosómico está reafirmando lo propuesto por Krapovickas (1960) al incluir a esta especie, junto a T. reichei, en una sección distinta, y plantea la posibilidad de que ambas especies en realidad correspondan a un género distinto y sin duda emparentado con Tarasa, sin embargo esta afirmación sólo podrá ser corroborada con el estudio cromosómico de $T$. reichei.

Una situación similar fue lo que ocurrió con Andeimalva J.A.Tate, donde un grupo de especies del género Tarasa aparecieron segregadas en un análisis filogenético, lo que además se veía respaldado por el número cromosómico básico $\mathrm{x}=6$, debido a lo cual se creó el género (Tate 2003b). Sin embargo, existen algunos géneros como Gaya Gaudin (Apiaceae) con especies con $2 n=12$ y una con $2 \mathrm{n}=14$ sin posibilidades de dividirlo en dos géneros, y como Arachis L. (Fabaceae), donde lo común es $2 n=20$ y 40 , no obstante hay 4 especies con $2 \mathrm{n}=18$, las que son muy difíciles de separar, siendo casi idénticas al resto (Krapovickas com. pers.).

Otros ejemplares fuera del área descrita no se han encontrado. La abundancia de zarzamora dificulta enormemente el acceso y una búsqueda más exhaustiva en sectores aledaños a lo largo del mismo bosque requiere varios días. Fue difícil determinar el número de individuos, ya que el hábito de crecimiento es muy intrincado, con esto no se puede descartar que se trate de un único ejemplar que se extiende en toda el área (unos 7 x 7 m) a través de un sistema de tallos semienterrados.

El sitio donde se encontró la planta en la actualidad es relativamente poco perturbado por actividades humanas, por lo que los ejemplares están relativamente protegidos de la intervención de personas y ganado. Sin embargo, la mayor amenaza está dada por el riesgo de incendio, evento que se ha registrado con anterioridad al menos una vez como consecuencia de quemas descuidadas en las parcelas del costado sur.

Debido a lo escaso de esta especie con un área de distribución restringida, se conoce sólo una localidad hasta ahora, y a las amenazas debidas a introducción de especies invasoras y peligro de incendios, lo que provoca una disminución en su calidad de hábitat, se considera a la especie dentro de la categoría En Peligro Crítico, CR B1a + B1b(iii) $+\mathrm{B} 2 \mathrm{a}+\mathrm{B} 2 \mathrm{~b}(\mathrm{iii})$, según los criterios de la UICN (2001). 

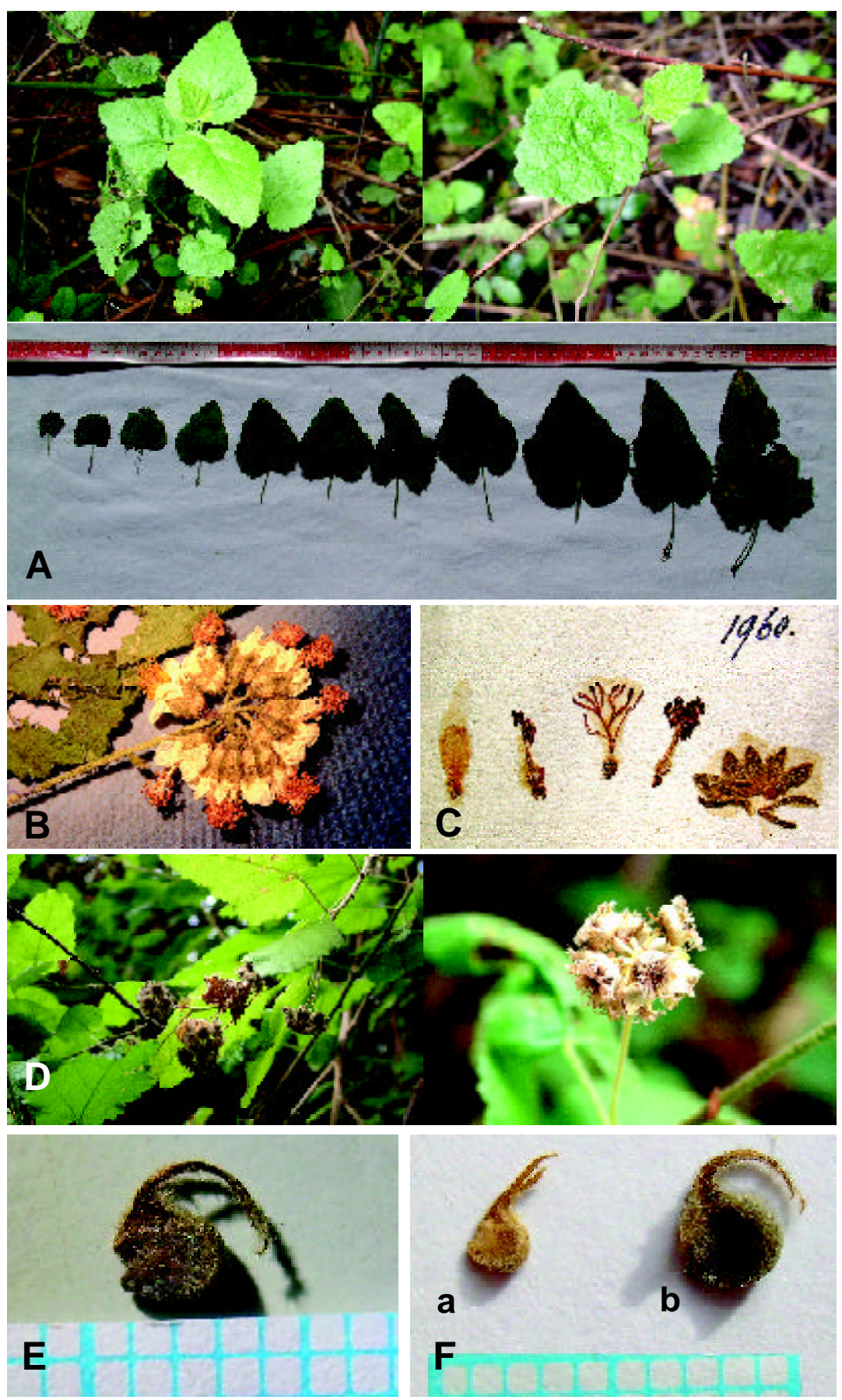

Figura 1. Tarasa umbellata. A. Variación de las hojas. B. Inflorescencia. C. Disección de una flor (SGO 41062). D. Frutos. E. Mericarpo. F. (a) Mericarpos de T. reichei (SGO 51946) y (b) T. umbellata (CONC 163806).

Figure 1. Tarasa umbellata. A. Leaves variation. B. Inflorescence. C. Dissection of a flower (SGO 41062). D. Fruits. E. Mericarp. F. (a) Mericarps of T. reichei (SGO 51946) and (b) T. umbellata (CONC 163806). 
Nuevo hallazgo de Tarasa umbellata. M ARTicorena, A. ET AL.

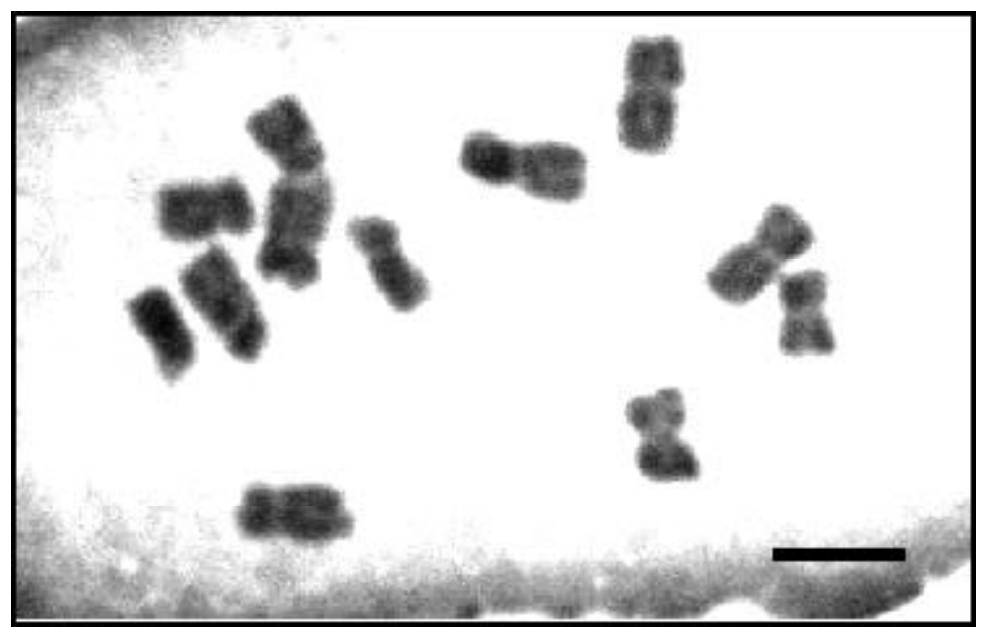

Figura 2. Metafase mitótica de Tarasa umbellata $2 \mathrm{n}=12$ (J.A. Valdivieso, CONC 163806). Barra: $3 \mu \mathrm{m}$.

Figure 2. Mitotic metaphase of Tarasa umbellata $2 \mathrm{n}=12$ (J.A. Valdivieso, CONC 163806). Bar: $3 \mu \mathrm{m}$.

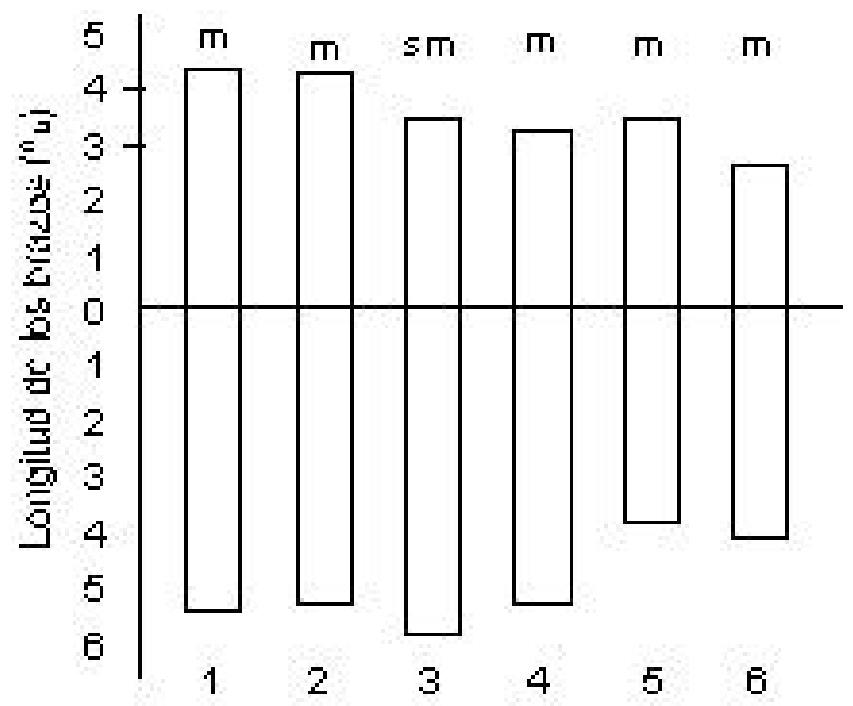

FIGURA 3. Idiograma del complemento cromosómico haploide de Tarasa umbellata $2 \mathrm{n}=12$ (J.A. Valdivieso, CONC 163806).

FIGURE 3. Idiogram of the haploid chromosome complement of Tarasa umbellata $2 \mathrm{n}=12$ (J.A. Valdivieso, CONC 163806). 


\section{AGRADECIMIENTOS}

Los autores agradecen a la Sra. Mélica Muñoz por el préstamo de material original del Museo Nacional de Historia Natural (SGO), al Ing. Antonio Kraprovickas por sus comentarios y sugerencias, y al Proyecto Flora de Chile.

\section{BIBLIOGRAFIA}

Krapovickas, A. 1954. Sinopsis del género Tarasa (Malvaceae). Boletín de la Sociedad Argentina de Botánica 5(3): 113-143.

Krapovickas, A. 1960. Poliploidía y área en el género Tarasa (Malvaceae). Lilloa 30: 233-249.

Levan, A., Fredga, K. \&A. SANDBERg. 1964. Nomenclature for centromeric position on chromosomes. Hereditas 52: 201-220.
Marticorena, A. 2005. Malvaceae (excepto Cristaria). En C. Marticorena y R. Rodríguez, Flora de Chile, Volumen 2, Fascículo 3: 22-105.

ReEves, A. 2001. MicroMeasure: a new computer program for the collection and analysis of cytogenetic data. Genome 44: 239-443.

Stewart, R.H. 2006. Malvaceae Info. Advice on Identification. http://www.malvaceae.info/ Identification/advice.html. Visitada: 11 abril 2007.

TAte, J. 2003a. Paraphyly of Tarasa (Malvaceae) and diverse origins of the polyploid species. Systematic Botany 28(4): 723-737.

TAтE, J. 2003b. Andeimalva, a new genus of Malvaceae from Andean South America. Lundellia 6:10-18.

UICN. 2001. Categories \& Criteria (version 3.1) http:// www.iucnredlist.org/info/categories_criteria2001 (Visitada: 31 Mayo de 2007).

Weiss-Schneeweiss, H., T.F. Stuessy, K. Tremetsberger, E. Urtubey, H. Valdebenito, S. Beck \& C. B aeza, 2007. Chromosome numbers and karyotypes of South American species and populations of Hypochaeris (Asteraceae). Botanical Journal of the Linnean Society 153: 49-60. 